\title{
openheart Intensified lipid-lowering treatment with alirocumab in patients with coronary heart disease
}

\author{
Daniel Steffens, ${ }^{1}$ Peter Bramlage (D) , ${ }^{2}$ Julia Müller, ${ }^{3}$ Cornelia Dorn, ${ }^{3}$ W Dieter Paar, ${ }^{3}$ \\ Celine Scheeff, ${ }^{1}$ Mario Kasner, ${ }^{1} \cup$ Rauch-Kröhnert ${ }^{1}$
}

To cite: Steffens D, Bramlage $P$ Müller J, et al. Intensified lipidlowering treatment with alirocumab in patients with coronary heart disease. Open Heart 2021;8:e001572. doi:10.1136/ openhrt-2021-001572

Received 5 January 2021 Revised 10 March 2021 Accepted 22 March 2021

\section{Check for updates}

(c) Author(s) (or their employer(s)) 2021. Re-use permitted under CC BY-NC. No commercial re-use. See rights and permissions. Published by BMJ.

${ }^{1}$ Klinik für Kardiologie, Campus Benjamin Franklin, Charité

- Universitätsmedizin Berlin, Berlin, Germany

Institut für Pharmakologie und Präventive Medizin GmbH, Cloppenburg, Germany ${ }^{3}$ Sanofi-Aventis Deutschland $\mathrm{GmbH}$, Berlin, Germany

Correspondence to Prof Dr U Rauch-Kröhnert; ursula.rauch@charite.de

\section{ABSTRACT}

Background Atherosclerotic cardiovascular disease is the leading cause of death and disability in the Western world. Objective To characterise adults with confirmed coronary heart disease (CHD) and primary heterozygous familial or non-familial hypercholesterolaemia or mixed dyslipidaemia who received alirocumab in a real-world setting.

Methods This open, prospective, multicentre, noninterventional study, conducted in Germany, enroled patients with confirmed CHD who were treated with alirocumab according to its summary of product characteristics. Prescription was at the physician's discretion and independent of study participation. Patients were followed for 12 weeks after alirocumab initiation. Results In total, 245 patients (mean age 62.2 years; $34.0 \%$ female) were documented at 90 sites. Overall, $47.7 \%$ had familial hypercholesterolaemia, $48.9 \%$ non-familial hypercholesterolaemia and $43.8 \%$ mixed dyslipidaemia; $74.6 \%$ had hypertension and $29.2 \%$ diabetes mellitus. The most common lipid-lowering therapy in the 12 months preceding alirocumab was a statin, often in combination with ezetimibe (73.5\%). Statin contraindications were documented for $46.2 \%$ patients and statin intolerance for $63.8 \%$. The mean low-density lipoprotein cholesterol (LDL-C)-level prior to alirocumab was $150.5 \pm 51.6 \mathrm{mg} / \mathrm{dL}$. Alirocumab prescription was in compliance with German national recommendations and/or European guidelines. The most common starting dose was $75 \mathrm{mg}$ every other week. Overall, $57 \%$ patients reached target LDL-C levels ( $<70 \mathrm{mg} / \mathrm{dL}$ ) after 12 weeks of treatment. Alirocumab was generally well tolerated. Conclusion In a real-world setting in Germany, alirocumab was prescribed for patients with atherosclerotic cardiovascular disease who had high baseline LDL-C levels with or without statin intolerance. Efficacy and safety were consistent with findings observed in the ODYSSEY Phase III programme.

\section{INTRODUCTION}

Atherosclerotic cardiovascular disease (ASCVD) is the leading cause of death and disability in the Western world ${ }^{1-3}$ and hypercholesterolaemia constitutes one of its major risk factors. Despite the availability of effective low-density lipoprotein cholesterol (LDL-C) lowering drugs, such as statins,

\section{Key questions}

What is already known about this subject?

- Atherosclerotic cardiovascular disease (ASCVD) is the leading cause of death and disability in the Western world and hypercholesterolaemia constitutes one of its major risk factors. Despite the availability of effective low-density lipoprotein cholesterol (LDL-C) lowering drugs (eg, statins), many individuals with familial hypercholesterolaemia (FH) or non-familial hypercholesterolaemia or mixed dyslipidaemia continue to have elevated LDL-C levels and remain at high risk for ASCVD.

What does this study add?

- This study provides data on the characteristics and treatment patterns of patients with established ASCVD who were prescribed alirocumab in daily clinical practice in Germany. Alirocumab was predominantly used in the working age population, with a predominance of male patients, and a pattern of metabolic comorbidity (eg, hypertension and/ or diabetes in addition to dyslipidaemia). FH was a frequent underlying disease. The efficacy and safety of alirocumab in this real-world setting were consistent with findings observed in the ODYSSEY Phase III programme.

How might this impact on clinical practice?

- The results of this study suggest that treatment with alirocumab can provide effective lipid-lowering in real-world conditions for patients with ASCVD who have high LDL-C levels with/without statin intolerance.

many individuals with familial hypercholesterolaemia $(\mathrm{FH})$ or non-familial hypercholesterolaemia or mixed dyslipidaemia continue to have elevated LDL-C values and, therefore, remain at high risk for ASCVD. ${ }^{24}$ Proprotein convertase subtilisin/kexin type 9 (PCSK9)inhibition is a new treatment strategy for patients who do not reach their LDL-C targets with conventional oral lipid-lowering treatment (LLT). Alirocumab, a fully human monoclonal antibody that binds with high affinity and specificity to PCSK9, has been 
evaluated in a large phase III clinical trial programme (ODYSSEY), consisting of 17 separate studies involving more than 24500 patients in total. A decrease in LDL-C of up to $60 \%$ was observed in these studies. ${ }^{5-7}$ The ODYSSEY OUTCOMES study demonstrated a reduction of recurrent ischaemic cardiovascular events in patients with a prior acute coronary syndrome and at high cardiovascular risk. ${ }^{69}$ PCSK9 inhibitors, however, need to be prescribed in the clinical and economical national environment which includes guideline recommendations of the European Society of Cardiology/European Atherosclerosis Society (ESC/EAS) ${ }^{10}$ and, for Germany, the Joint Federal Committee (G-BA) recommendations.

At the time of patient recruitment, the ESC/EAS task force on PCSK9 inhibitors ${ }^{9}$ recommended treatment with PCSK9-inhibitors for patients with clinical ASCVD and substantially elevated LDL-C levels despite being on maximally tolerated statin therapy (with or without ezetimibe). They were further recommended for patients with ASCVD who are unable to tolerate at least three statins, and for patients with FH without clinical ASCVD but with substantially elevated LDL-C levels despite treatment with statins plus ezetimibe. In the 2019 guideline revision, ${ }^{10}$ PCSK9 inhibitors are recommended for very high-risk patients in secondary prevention (class I, level A), for very high-risk patients with FH (IC) and could be considered in very high-risk patients without $\mathrm{FH}$ (IIbC), given that they do not achieve their treatment goals on maximum tolerated doses of a statin and ezetimibe. They are also considered a combination partner for ezetimibe, if statins are not tolerated (IIbC).

The G-BA comprises a group of German public health agencies that is authorised to make binding decisions for millions of people in the public health insurance system, based on reform bills passed by lawmakers along with routine decisions regarding healthcare in Germany. Government officials are responsible for exercising legal supervision over the committee's decisions and guidelines. The G-BA has determined that alirocumab can be prescribed in patients with heterozygous $\mathrm{FH}$, non-FH or mixed dyslipidaemia with treatment-refractory courses in which, despite maximum dietary efforts and LLT (statins and/or other lipid-lowering agents in case of statin contraindications) documented over a period of 12 months, LDL-C cannot be lowered sufficiently and it is therefore assumed that the indication to perform LDL apheresis exists. Only patients with established, progressive vascular disease (coronary heart disease (CHD), cerebrovascular manifestation, peripheral artery disease $(\mathrm{PAD})$ ) and other risk factors (eg, diabetes mellitus, kidney function glomerular filtration rate $<60 \mathrm{~mL} / \mathrm{min}$ or New York Heart Association (NYHA) heart failure III and IV) are eligible. The drug may only be prescribed by cardiologists, nephrologists, diabetologists, endocrinologists or specialists working in outpatient departments for lipid metabolism disorders.

The clinical and economical guidelines differ with respect to their eligibility criteria, such as the sole reliance on statin failure (G-BA), the definition of the length of the prior attempt (G-BA 12 months), the notion that PCSK9 inhibitors can only be prescribed in patients who would otherwise be considered to have an indication for LDL apheresis (G-BA), the omission of primary prevention patients (G-BA) and the physician type allowed to make the prescription. Considering the different recommendations, it remains unclear how these partially conflicting recommendations are applied in clinical practice. Hence, we aimed to study the patient characteristics and treatment patterns under these conditions and document the clinical effectiveness and safety of alirocumab under real-world conditions in Germany.

\section{METHODS}

OPTIMIZE was a national (German) prospective, openlabel, multicentre, non-interventional study conducted according to the German Drug Law ( $\$ 4$ part 23) in 90 centres in Germany ('Nicht-interventionelle Studie'; study number 433). ${ }^{11}$ Study sites were distributed across the country to ensure the data gathered were representative of patients treated with alirocumab throughout Germany.

Patients with confirmed CHD were included that were treated with alirocumab according to its summary of product characteristics (SmPC). According to the German Regulation for non-interventional studies, patients could only be included after the decision to intensify LLT was made by the treating physician according to the SmPC and G-BA regulation.

The study was performed in accordance with the ethical principles outlined in the Declaration of Helsinki, and all applicable amendments laid down by the World Medical Assemblies and the International Conference Harmonization guidelines for Good Clinical Practice. The study protocol was approved by the local institutional review board and independent ethics committee at each site. All patients provided written informed consent prior to documentation.

\section{Patient population}

The study included adult patients ( $\geq 18$ years) treated with alirocumab in accordance with the SmPC (Sanofi Aventis Deutschland GmbH, Frankfurt, Germany) and the G-BA recommendations as outlined above. This constituted a diagnosis of primary hypercholesterolaemia (heterozygous familial or non-familial) or mixed dyslipidaemia, and confirmed CHD with an acute coronary syndrome as defined by ODYSSEY OUTCOMES, ${ }^{689}$ and documented maximum dietary or LLT within the last 12 months. Contraindications to statins were identified by the treating physician. Statin intolerance was defined as the intolerance of at least two different statins due to unexplained skeletal muscle-related symptoms (eg, pain, pulling, weakness or cramps) that began or worsened during statin therapy and were reversible after discontinuation. A distinction was made between total statin 
intolerance due to which the patient no longer received statin and partial statin intolerance. With a partial intolerance, the patient could only tolerate a limited statin dose, which did not lead to the achievement of his/her individual target value.

\section{Data collection}

The observational period for each patient was 12 weeks, including a baseline visit (V1) at the time of alirocumab initiation, and one follow-up visit (V2) in accordance with routine clinical practice. Patient data were documented on paper-based case report forms. Adverse events (AEs) were reported from the time the physician obtained the patient's informed consent until 7 days after the end of the observation period (V2). Quality control measures included on-site visits at selected study centres $(>5 \%$ of all 90 centres), including source data verification by interview technique.

\section{Objectives}

The primary objective was a descriptive analysis of LLT in adult patients with primary hypercholesterolaemia (heterozygous familial or non-familial) or mixed dyslipidaemia and accompanied by dietary measures. This included treatment (1) in combination with a statin with or without other LLT in patients who did not reach their LDL-C goal despite being on the maximum tolerated statin dose or (2) as monotherapy or in combination with other LLTs in patients with intolerance of, or contraindications for, statins and (c) in patients with confirmed CHD.

It included a descriptive analysis of patient characteristics relevant to the German health insurance reimbursement criteria, including: (1) prior LLT within the last 12 months, (2) prior dietetic and lifestyle changes, (3) proportion of patients with heterozygous FH, (4) proportion of patients with mixed dyslipidaemia and (e) a description of any CHD.

Secondary objectives comprised: (1) evaluation of the efficacy of alirocumab as measured by the LDL-C lowering effect after approximately 12 weeks; (2) the identification of possible $\mathrm{FH}$ based on the procedure proposed by Klose et $a l^{12}$; and (3) analysis of patients with a possible statin intolerance.

\section{Statistics}

Due to the non-interventional observational study design, no formal sample size was determined. Quantitative data (eg, age) were analysed using means and SD or medians with IQRs, as appropriate. Qualitative data (eg, gender) were presented as proportions. 95\% CIs were provided, if appropriate, for qualitative and quantitative data. Safety was analysed among all patients receiving at least one alirocumab dose (safety analysis set, SAS). Descriptive and efficacy variables were analysed in the full analysis set (FAS), which included all patients in SAS who met the selection criteria and had sufficient data for the primary analysis (at least one LDL-C value at baseline and on treatment). All statistical analyses were carried out using the SAS package (V.9.4, Cary, North Carolina, USA).

\section{RESULTS}

In total, 245 patients from 90 sites were documented (figure 1). As one patient received no dose of alirocumab, the SAS consisted of 244 patients; 200 patients (from 77

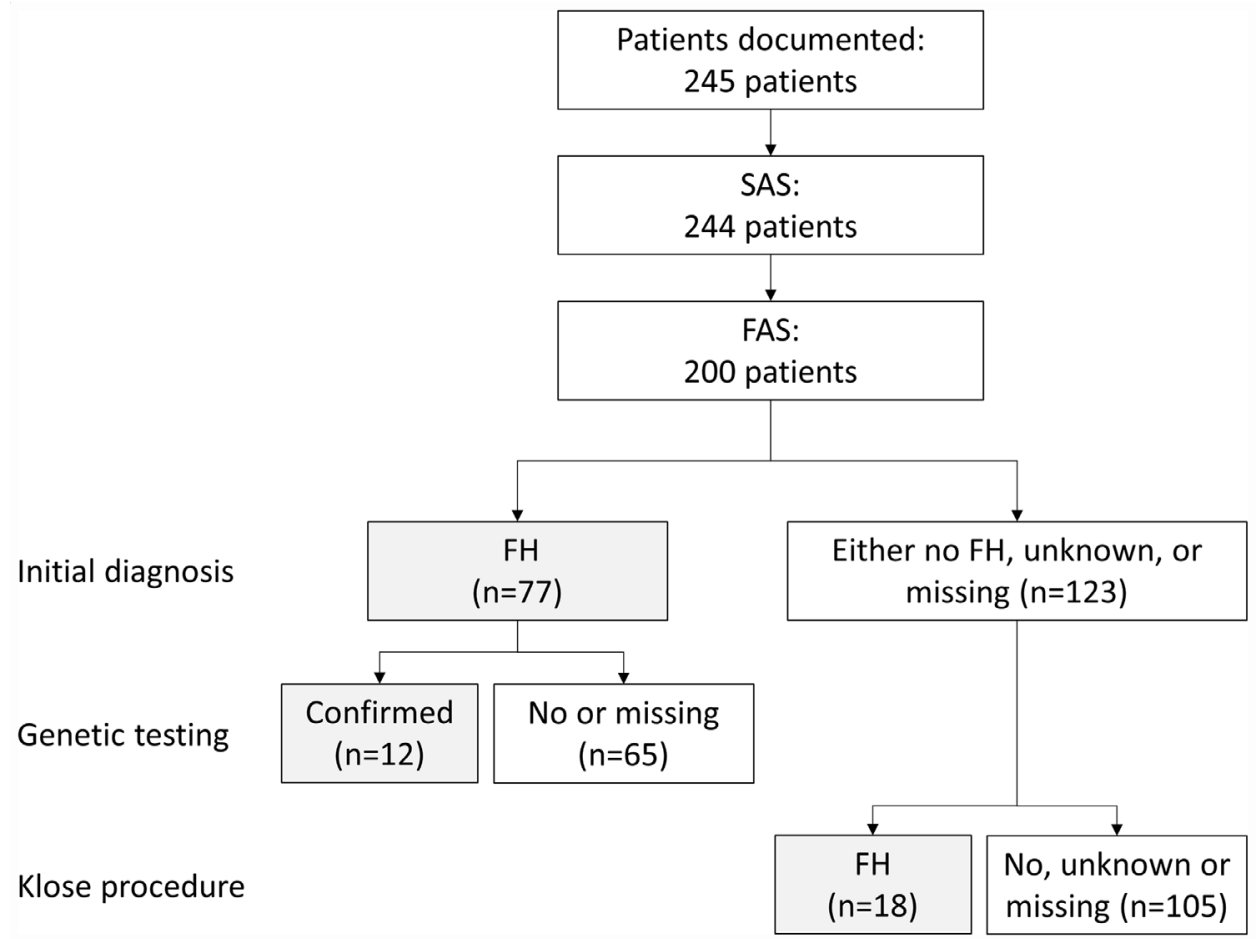

Figure 1 Patient flow and diagnosis of FH. FAS, full analysis set; FH, familial hypercholesterolaemia; SAS, safety analysis set. 


\begin{tabular}{|c|c|}
\hline & $\mathbf{N}(\%)$ or mean \pm SD \\
\hline Age (years) & $62.2 \pm 10.5$ \\
\hline Female gender & $68(34.0)$ \\
\hline Weight (kg) & $84.9 \pm 15.1$ \\
\hline BMI $\left(k g / m^{2}\right)(n=198)$ & $28.5 \pm 4.5$ \\
\hline$<18.5 \mathrm{~kg} / \mathrm{m}^{2}$ & $0(0)$ \\
\hline 18.5 to $<25 \mathrm{~kg} / \mathrm{m}^{2}$ & $45(22.7)$ \\
\hline 25 to $<30 \mathrm{~kg} / \mathrm{m}^{2}$ & $90(45.5)$ \\
\hline$\geq 30 \mathrm{~kg} / \mathrm{m}^{2}$ & $63(31.8)$ \\
\hline Systolic BP (mm Hg) & $131.5 \pm 14.9$ \\
\hline Diastolic BP (mm Hg) & $78.2 \pm 8.9$ \\
\hline \multicolumn{2}{|l|}{ Dyslipidaemia } \\
\hline $\mathrm{FH}$ & $95(47.7 ; 95 \% \mathrm{Cl} 40.6$ to 54.9$)$ \\
\hline $\begin{array}{l}\text { Non-familial } \\
\text { hypercholesterolaemia }\end{array}$ & $93(48.9 ; 95 \% \mathrm{Cl} 41.6$ to 56.3$)$ \\
\hline Mixed dyslipidaemia & $84(43.8 ; 95 \% \mathrm{Cl} 36.6$ to 51.1$)$ \\
\hline $\begin{array}{l}\text { None of the above or missing } \\
\text { information }\end{array}$ & $9(4.5 ; 95 \% \mathrm{Cl} 2.1$ to 8.4$)$ \\
\hline Statin contraindication $(n=195)$ & $90(46.2)$ \\
\hline Statin intolerance $(n=199)$ & $127(63.8)$ \\
\hline \multicolumn{2}{|l|}{ Cardiovascular disease } \\
\hline Any cardiovascular disease & $200(100)$ \\
\hline $\mathrm{CHD}$ & $197(98.5)$ \\
\hline Status post ACS & $169(84.5)$ \\
\hline Hypertension & $147(74.6)$ \\
\hline Heart failure & $29(15.5)$ \\
\hline PAD & $26(13.7)$ \\
\hline Stroke & $17(8.9)$ \\
\hline Transient ischaemic attack & $7(3.7)$ \\
\hline \multicolumn{2}{|l|}{ Diabetes mellitus ( $n=195)$} \\
\hline Type 1 & $6(3.1)$ \\
\hline Type 2 & $51(26.2)$ \\
\hline $\begin{array}{l}\text { Disease management programme } \\
(\mathrm{n}=197)\end{array}$ & $45(22.8)$ \\
\hline
\end{tabular}

ACS, acute coronary syndrome; BMI, body mass index; BP, blood pressure; $\mathrm{CHD}$, coronary heart disease; FAS, full analysis set; $\mathrm{FH}$, familial hypercholesterolaemia; PAD, peripheral artery disease.

sites) met all selection criteria and had sufficient data for the primary analysis and were included in the FAS.

\section{Patient characteristics}

The mean age of the 200 patients in the FAS was 62.2 years and $68(34.0 \%)$ were female (table 1$)$. The mean body mass index was $28.5 \mathrm{~kg} / \mathrm{m}^{2}$. A total of $95(47.7 \%)$ patients suffered from FH, 93 (48.9\%) patients had non-familial hypercholesterolaemia and $84(43.8 \%)$ patients had mixed dyslipidaemia (non-exclusive). In accordance with the patient selection criteria, the most common comorbid condition was CHD ( $\mathrm{n}=197,98.5 \%)$. Furthermore, 169
$(84.5 \%)$ patients had documented prior acute coronary syndrome (ACS), 147 (74.6\%) patients had hypertension and $29.2 \%$ of patients (57 of 195 with data) had diabetes mellitus.

With respect to patients with $\mathrm{FH}$, initially 77 (38.5\%) patients in the FAS were diagnosed with FH (figure 1), of whom $12(15.5 \%)$ were confirmed by genetic diagnostics. Applying the algorithm suggested by Klose et al, ${ }^{12}$ to the 123 patients initially described as having no $\mathrm{FH} /$ unknown FH status/missing data, identified a further 18 (14.6\%) patients with FH.

\section{Lipid-lowering therapy (LLT)}

During the 12 months prior to alirocumab initiation (in which statins may have been replaced over time by another), the most common LLT (alone or in combination therapy) was atorvastatin, which was prescribed for $72.0 \%$ of the patients (mean dose $48.3 \mathrm{mg} /$ day), followed by simvastatin $(41.0 \%$; mean dose $36.7 \mathrm{mg} /$ day) and rosuvastatin $(22.0 \%$; mean dose $16.4 \mathrm{mg}$ /day, table $2 \mathrm{~A}$ ). Most patients received these statins in combination with ezetimibe $(73.5 \%)$, with a mean achieved LDL-C of $143.0 \mathrm{mg} / \mathrm{dL}$ (95\% CI 119.9 to 166.1 ). When alirocumab was initiated (table 2B, at baseline) atorvastatin was used in parallel in $40.5 \%$ cases, rosuvastatin in $12.0 \%$ and statin/ezetimibe combinations in $32.5 \%$. From baseline to the 12-week visit, the use of statins varied (eg, rosuvastatin increased from $12.0 \%$ to $16.2 \%$ ) and ezetimibe from $45.0 \%$ to $50.0 \%$.

A statin contraindication was documented for $46.2 \%$ (95\% CI 39.0 to 53.4) of the patients and a statin intolerance for $63.8 \%$ (95\% CI 56.7 to 70.5 ). The three most frequent reasons for the diagnosis of statin intolerance were myalgia without creatine kinase elevation $(n=73$, $57.5 \%)$, muscle disorders $(\mathrm{n}=49,38.6 \%)$, and oversensitivity/others ( $\mathrm{n}=26,20.5 \%)$.

Prior to starting alirocumab, 127 patients had not reached their LDL-C goal despite being treated with the maximum tolerated statin dose. At baseline, 26 (20.5\%) of these patients were prescribed alirocumab alone, 60 $(47.2 \%)$ alirocumab in combination with a statin, 21 $(16.5 \%)$ alirocumab in combination with other LLT and $20(15.7 \%)$ alirocumab in combination with both a statin and another LLT (table 3). Among 128 patients with statin intolerance or contraindication, $28.9 \%$ were prescribed alirocumab as monotherapy, $40.6 \%$ in combination with a statin, $22.7 \%$ in combination with other LLT and $7.8 \%$ in combination with both a statin and another LLT.

\section{Alirocumab treatment}

The most common dosage of alirocumab at the start of therapy was $75 \mathrm{mg}$ every other week $(\mathrm{n}=122 ; 61.3 \%$, figure 2). After 12 weeks, the most common dose used was $150 \mathrm{mg}$ every other week $(\mathrm{n}=101 ; 54.3 \%)$. No patients received $300 \mathrm{mg}$ at 4 -week intervals (another dosing regimen in the SmPC). Most patients $(n=151$; $81.2 \%$ ) remained on their starting dose; the dose 
Coronary artery disease

Table 2A Concomitant LLT in the 12 months preceding initiation of treatment with alirocumab (FAS; $n=200)^{\star}$

\begin{tabular}{|c|c|c|c|}
\hline & LLT durir & efore baseline & \\
\hline & $\mathbf{N}(\%)$ & $\begin{array}{l}\text { Daily dose }(\mathrm{mg}) \\
\text { mean }(95 \% \mathrm{Cl})\end{array}$ & $\begin{array}{l}\text { LDL-C on max dose }(\mathrm{mg} / \mathrm{dL}) \\
\text { mean }(95 \% \mathrm{Cl})\end{array}$ \\
\hline Atorvastatin $\dagger$ & $144(72.0)$ & 48.3 (44.0 to 52.5$)$ & 152.6 (126.1 to 179.1$)$ \\
\hline Simvastatin† & $82(41.0)$ & 36.7 (32.2 to 41.2$)$ & 164.5 (146.2 to 182.8$)$ \\
\hline Rosuvastatin $†$ & $44(22.0)$ & 16.4 (12.2 to 20.6$)$ & 119.0 (105.5 to 132.4$)$ \\
\hline Fluvastatin† & 27 (13.5) & 33.1 (26.0 to 40.2 ) & $140.4(130.4$ to 150.5$)$ \\
\hline Pravastatin $†$ & $21(10.5)$ & 25.4 (19.2 to 31.6$)$ & $133.9(113.0$ to 154.9$)$ \\
\hline Ezetimibe & $1(0.5)$ & 10.0 & 129.0 \\
\hline Any statin + Ezetimibe & $147(73.5)$ & n.a. & 143.0 (119.9 to 166.1$)$ \\
\hline Other/noneł & $11(5.5)$ & n.a. & n.a. \\
\hline
\end{tabular}

The numbers reflect a 12-month period with potential switches between statins. Thus, the numbers are much higher than if you look at a single timepoint, eg, baseline.

*Potentially time-displaced for the 12 months prior to inclusion.

†Alone or in combination.

†Including colesevelam hydrochloride.

FAS, full analysis set; LDL-C, low density lipoprotein cholesterol; LLT, lipid-lowering therapy.

Table 2B Concomitant LLT at baseline and at end of follow-up (FAS; $n=200)^{*}$

\begin{tabular}{|c|c|c|}
\hline & LLT at baseline & LLT at 12 weeks \\
\hline & $N(\%)$ & N (\%) \\
\hline Alirocumab* & $200(100.0)$ & $198(100.0)$ \\
\hline Atorvastatin* & $81(40.5)$ & $85(42.9)$ \\
\hline Simvastatin* & $14(7.0)$ & $20(10.1)$ \\
\hline Rosuvastatin* & $24(12.0)$ & $32(16.2)$ \\
\hline Fluvastatin* & $5(2.5)$ & $11(5.5)$ \\
\hline Pravastatin* & $4(2.0)$ & $9(4.5)$ \\
\hline Ezetimibe $^{*}$ & $90(45.0)$ & $99(50.0)$ \\
\hline Othert & $10(5.0)$ & $17(8.6)$ \\
\hline None & $0(0.0)$ & $0(0.0)$ \\
\hline Any alirocumab combination & $157(78.5)$ & $162(81.8)$ \\
\hline Any statin + Ezetimibe & $65(32.5)$ & $74(37.4)$ \\
\hline Any other combination & $0(0.0)$ & $0(0.0)$ \\
\hline
\end{tabular}

${ }^{*}$ Alone or in combination.

†Including colesevelam hydrochloride.

FAS, full analysis set; LDL-C, low density lipoprotein cholesterol; LLT, lipid-lowering therapy.

was up-titrated in $17.2 \%(\mathrm{n}=32)$ and down-titrated in $1.6 \%(\mathrm{n}=3)$ of patients.

At baseline, average lipid levels were as follows: LDL-C $150.5 \mathrm{mg} / \mathrm{dL}$, total cholesterol $226.9 \mathrm{mg} /$ dL, high-density lipoprotein cholesterol $48.4 \mathrm{mg} /$ $\mathrm{dL}$, triglycerides $204.9 \mathrm{mg} / \mathrm{dL}$, and lipoprotein(a) $62.1 \mathrm{mg} / \mathrm{dL}$ (figure 3). At the 12-week follow-up, LDL-C was significantly reduced by $-78.6 \mathrm{mg} / \mathrm{dL}$ $(95 \%$ CI -86.7 to -70.5$)$ to a final value of $76.3 \mathrm{mg} / \mathrm{dL}$, which constituted a target achievement of $<70 \mathrm{mg} / \mathrm{dL}$ in $57.0 \%$ of patients. This was accompanied by reductions in other lipid values, including total cholesterol $(-82.7 \mathrm{mg} / \mathrm{dL} ; 95 \% \mathrm{CI}-91.4$ to -71.4$)$, triglycerides $(-45.4 \mathrm{mg} / \mathrm{dL} ; 95 \% \mathrm{CI}-78.4$ to -12.4$)$ and lipoprotein (a) $(-18.4 \mathrm{mg} / \mathrm{dL} ; 95 \% \mathrm{CI}-31.0$ to -5.8$)$.
Overall, $76.5 \%(\mathrm{n}=153)$ patients treated with alirocumab met one of the ESC/EAS guideline criteria for prescribing alirocumab (table 4). All patients met at least one of the G-BA recommendations for the selection of patients for PCSK9 inhibitor treatment.

\section{Adverse events}

In total, $11 \mathrm{AEs}$ were reported among 4 (1.6\%) patients out of the 244 patients of the SAS population. Seven of these recovered and four were ongoing at the end of the study. These ongoing AEs affected two patients with (1) pruritic rash and (2) myalgia/arthralgia, fatigue, headache. No serious AEs occurred. AEs led to study drug withdrawal for three $(1.2 \%)$ patients, 
Table 3 Descriptive analysis of LLT at baseline, and mean change after 12 weeks of follow-up, according to type of patient

\begin{tabular}{|c|c|c|c|c|}
\hline \multirow{3}{*}{$\begin{array}{l}\text { Patients not at their LDL-C goal on maximum tolerated } \\
\text { dose of LLT at baseline }(n=127)\end{array}$} & \multicolumn{2}{|l|}{ Baseline } & \multirow{2}{*}{$\begin{array}{l}12 \text { weeks } \\
\text { Mean LDL-C } \\
\text { change } \pm S D \\
(\mathrm{mg} / \mathrm{dL})\end{array}$} & \multirow[b]{3}{*}{$95 \% \mathrm{Cl}$} \\
\hline & n (\%) & n (\%) & & \\
\hline & $\mathrm{n}=127$ & $\mathrm{n}=127$ & $\mathrm{n}=91$ & \\
\hline Alirocumab monotherapy & $26(20.5)$ & $23(18.1)$ & $-48.0 \pm 14.1$ & -57.5 to -37.7 \\
\hline Alirocumab + statin & $60(47.2)$ & $51(40.2)$ & $-50.0 \pm 23.2$ & -67.9 to -35.0 \\
\hline Alirocumab + other LLT & $21(16.5)$ & $21(16.5)$ & $-56.3 \pm 11.4$ & -62.7 to -45.5 \\
\hline Alirocumab + statin + other LLT & $20(15.7)$ & $32(25.2)$ & $-53.8 \pm 17.5$ & -62.7 to -40.3 \\
\hline Patients with statin intolerance or contraindication at baseline $(n=128)$ & $n=128$ & $n=128$ & $\mathrm{n}=64$ & \\
\hline Alirocumab monotherapy & $37(28.9)$ & $32(25.0)$ & $-48.2 \pm 13.4$ & -57.4 to -40.4 \\
\hline Alirocumab + statin & $52(40.6)$ & $45(35.2)$ & $-43.2 \pm 22.4$ & -58.9 to -28.5 \\
\hline Alirocumab + other LLT & $29(22.7)$ & $30(23.4)$ & $-56.3 \pm 11.4$ & -62.7 to -45.5 \\
\hline Alirocumab + statin + other LLT & $10(7.8)$ & $21(16.4)$ & $-45.0 \pm 10.2$ & -51.6 to -39.4 \\
\hline $\begin{array}{l}\text { Patients not at goal or with statin intolerance/contraindication at baseline } \\
(\mathrm{n}=175)\end{array}$ & $\mathrm{n}=175$ & $\mathrm{n}=175$ & $\mathrm{n}=94$ & \\
\hline Alirocumab monotherapy & $41(23.4)$ & $36(20.6)$ & $-48.3 \pm 13.3$ & -57.4 to -40.4 \\
\hline Alirocumab + statin & $82(46.9)$ & $71(40.6)$ & $-50.3 \pm 23.0$ & -66.9 to -36.2 \\
\hline Alirocumab + other LLT & $29(16.6)$ & $30(17.1)$ & $-56.3 \pm 11.4$ & -62.7 to -45.5 \\
\hline Alirocumab + statin + other LLT & $23(13.1)$ & $38(21.7)$ & $-53.8 \pm 17.5$ & -62.7 to -40.3 \\
\hline
\end{tabular}

LDL-C, low density lipoprotein cholesterol; LLT, lipid-lowering therapy.

with the event considered related to the study drug for two of these patients (table 5).

\section{DISCUSSION}

This study provides data on the characteristics and treatment patterns of patients with established ASCVD who were prescribed alirocumab in daily clinical practice in Germany. Our study showed a male predominance, which for the population under investigation is not unusual. In the vast majority of federal states in Germany, the lifetime prevalence of major cardiovascular disease (myocardial infarction, other CHD, stroke and congestive heart failure) is higher in men compared with women. ${ }^{13}$ Furthermore, women tend to manifest CHD later in life than males and it is possible, therefore, that the female subjects in our study population was too 'young' for symptoms of CHD. ${ }^{14}$

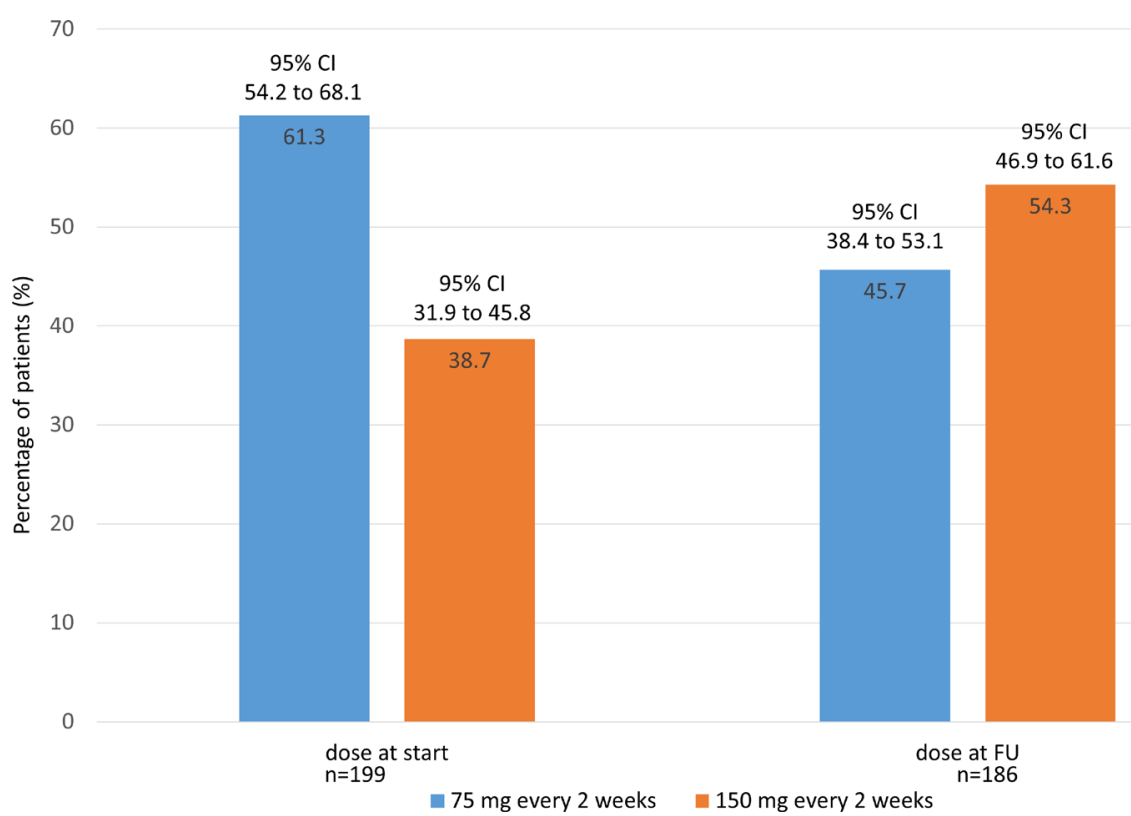

Figure 2 Dose of alirocumab at baseline and 12 week follow-up (FAS population). FAS, full analysis set. 


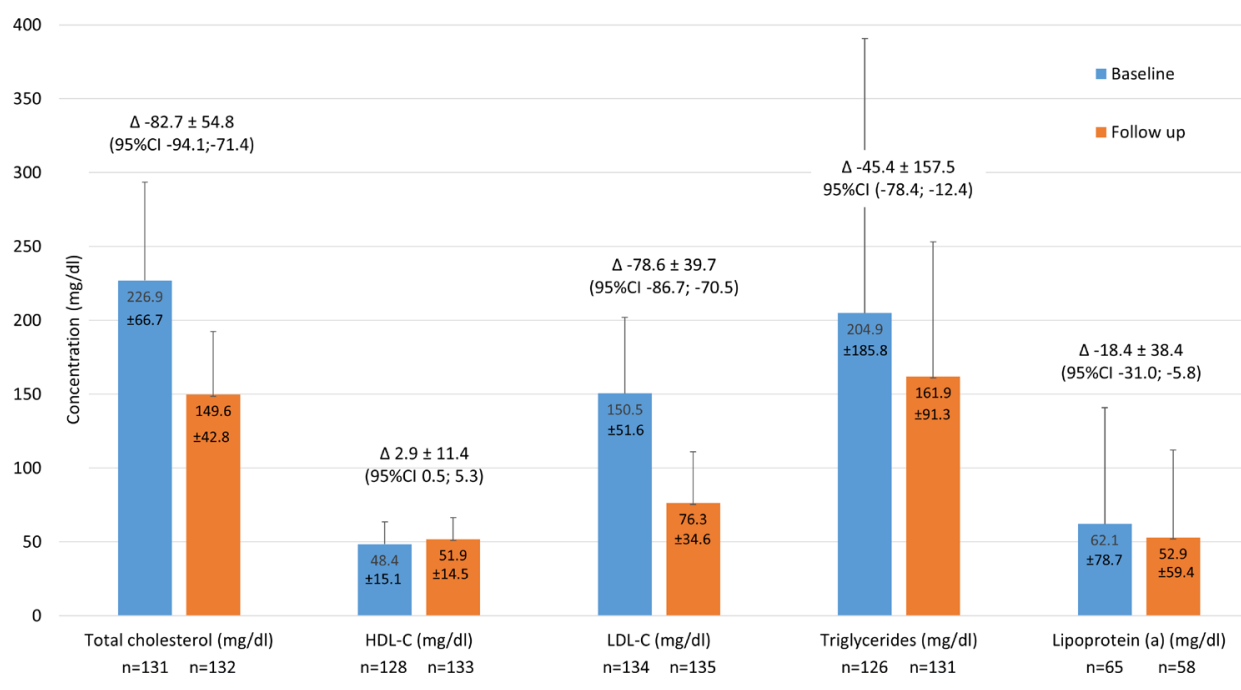

Figure 3 Lipid profiles at baseline and 12 week follow-up (FAS population). $\Delta$, mean (SD) per cent change from baseline to week 12; HDL-C, high-density lipoprotein cholesterol; LDL-C, low-density lipoprotein cholesterol.

\begin{tabular}{|c|c|}
\hline ESC/EAS guideline criteria $^{9}$ & $\mathrm{n} / \mathrm{N}(\%)$ \\
\hline ASCVD and LDL-C not at goal \& max. tolerated statin dose & $79 / 200(39.5)$ \\
\hline ASCVD and statin intolerance (three statins) & $127 / 200(63.5)$ \\
\hline FH and LDL-C not at goal (individual target) & $60 / 200(30.0)$ \\
\hline Any of the above & $153 / 200(76.5)$ \\
\hline G-BA recommendation & $\mathrm{n} / \mathrm{N}(\%)$ \\
\hline \multicolumn{2}{|l|}{ Any of } \\
\hline $\mathrm{FH}$ & $95 / 199(47.7)$ \\
\hline Non-familial hypercholesterolaemia & $93 / 190(48.9)$ \\
\hline Mixed dyslipidaemia & $84 / 192(43.8)$ \\
\hline \multicolumn{2}{|l|}{ Plus any of } \\
\hline Maximum dietary effort & $191 / 191(100.0)$ \\
\hline LDL-C not at goal (individual target) & $53 / 91(58.2)$ \\
\hline Statin contraindication & $86 / 187(46.0)$ \\
\hline \multicolumn{2}{|l|}{ Plus any of } \\
\hline CHD, cerebrovascular disease, PAD or ACS & 191/191 (100.0) \\
\hline CV risk factor (diabetes, renal dysfunction) & $68 / 191(35.6)$ \\
\hline FH (only) but no further disease or risk & $0 / 191(0.0)$ \\
\hline Number of those with at least one of the three categories each & 191 \\
\hline
\end{tabular}

ACS, acute coronary syndrome; ASCVD, atherosclerotic cardiovascular disease; CHD, coronary heart disease; CV, cardiovascular; EAS, European Atherosclerosis Society; ESC, European Society of Cardiology; FH, familial hypercholesterolaemia; LDL-C, low-density lipoprotein cholesterol; PAD, peripheral artery disease.

Alirocumab was predominantly used in the working age population, with a predominance of male patients, and a pattern of metabolic comorbidity (eg, hypertension and/or diabetes in addition to dyslipidaemia). FH was a frequent underlying disease. When the algorithm suggested by Klose et $a l^{12}$ was applied to the study population, additional patients with $\mathrm{FH}$ were identified among those originally considered to have other diagnoses. Patients with FH and elevated LDL-C levels despite maximally tolerated statin plus ezetimibe therapy are considered suitable candidates for treatment with a PCSK9 inhibitor. ${ }^{9}$ The proportion of patients prescribed alirocumab who had $\mathrm{FH}$ in the current study $(47.7 \%)$ is consistent with that reported in another observational study of alirocumab prescribed in routine clinical practice in Germany

Table 5 Summary of safety data (SAS, $n=244)$

\begin{tabular}{|ll} 
Patients with at least one & $\begin{array}{l}\text { Number of } \\
\text { patients } \\
\text { (\%) }\end{array}$ \\
\hline AE & $4(1.6)$ \\
AE leading to study withdrawal & $3(1.2)$ \\
Drug-related AE & $2(0.8)$ \\
Non-serious AE & $4(1.6)$ \\
\hline
\end{tabular}

Type of AE Number of
events (\%)

Nervous system disorders

\begin{tabular}{ll} 
Headache & $2(0.8)$ \\
Syncope & $1(0.4)$ \\
Musculoskeletal and connective tissue disorders & \\
$\quad$ Myalgia & $2(0.8)$ \\
Arthralgia & $1(0.4)$ \\
$\quad$ Neck pain & $1(0.4)$ \\
Cardiac disorders & \\
$\quad$ Tachycardia & $1(0.4)$ \\
General disorders and administration site conditions & \\
$\quad$ Fatigue & $1(0.4)$ \\
Psychiatric disorders & \\
$\quad$ Depressive symptom & $1(0.4)$ \\
Skin and subcutaneous tissue disorders & $1(0.4)$ \\
\hline Rash pruritic &
\end{tabular}

Multiple entries possible.

$A E$, adverse event; SAS, safety analysis set. 
$(50.8 \%)$, but slightly lower than that reported for a UK study (66\%).

Prior to initiating alirocumab, treatment with statins (particularly atorvastatin, simvastatin and rosuvastatin) was generally used to try and control the dyslipidaemia, either alone or, more frequently, in combination with ezetimibe. This would be in accordance with guideline recommendations to use statins as first-line therapy in patients with hypercholesterolaemia, with the addition of ezetimibe if necessary. ${ }^{4}$ Among the study population, many $(63.5 \%)$ patients had not reached their LDL-C goal on their previous treatment, which included a maximally tolerated statin dose. In addition, a substantial number of patients prescribed alirocumab were considered to have a statin contraindication $(46 \%)$ or not to tolerate high statin doses $(64 \%)$. Both of these reasons would make patients suitable candidates for treatment with a PCSK9 inhibitor according to ESC/EAS guidance. ${ }^{9}$ A recent simulation study suggested that, despite maximised statin and ezetimibe therapy, approximately $50 \%$ of patients with a recent myocardial infarction would be eligible for PCSK9 inhibitor therapy based on ESC/EAS recommendations. ${ }^{16}$ An observational study in German clinical practice with the same underlying definition of statin intolerance as in our study found that $72.8 \%$ of patients prescribed alirocumab had statin intolerance, while a UK study reported a rate of $82 \%$. The higher percentage of patients in the UK study might be explained by a slightly different definition of statin intolerance. They declared patients as intolerant to statin with intolerance to one or more statins since diagnosis. In our study, it was intolerance to at least two statins. All patients prescribed alirocumab in the current study complied with the G-BA and/ or the ESC/EAS guideline criteria. In particular, a high level of compliance with the G-BA recommendations was observed, suggesting physicians prioritised meeting national requirements over following international guidelines.

During treatment with alirocumab, statin comedication was common, and even triple combinations were used in some patients. Except for patients with a statin contraindication/intolerance, it would be recommended to add a PCSK9 inhibitor to existing statin therapy (with or without ezetimibe).$^{79}$ Alirocumab was most often initiated at a dose of $75 \mathrm{mg}$ every other week ( $>60 \%$ of patients), and the dose was increased to $150 \mathrm{mg}$ every other week in $17.2 \%$ of patients by week 12 . These dosages are consistent with the SmPC for alirocumab. Another observational study in Germany reported that approximately $73 \%$ of patients prescribed alirocumab received an initial dose of $75 \mathrm{mg}$, and that the dose was subsequently increased in almost $20 \%$ of patients. Similar findings were reported for a UK study ( $72 \%$ and $23.3 \%$, respectively). Although many patients in the current study had failed to achieve target LDL-C levels on their previous LLT, more than half were able to reach their individual or guideline-based target (LDL-C $<70 \mathrm{mg} / \mathrm{dL}$ ) with alirocumab treatment.
This target is recommended by guidelines to reduce the risk of recurrent cardiovascular events. ${ }^{4}$ The reduction was even achieved in the absence of any specific guidance on up-titration or a target dose. The reductions in LDL-C levels achieved with alirocumab in the current study are consistent with those seen in other real-world studies of alirocumab and other PCSK9 inhibitors. ${ }^{17-20}$

Alirocumab (with or without other LLT) appeared to be generally well tolerated during the observation period. Study drug was withdrawn in three patients, and two patients had ongoing AEs at the end of the 12-week observation period, including pruritic rash in one patient and myalgia/arthralgia, fatigue and headache in the other. Alirocumab has also been found to be generally well tolerated in other real-world studies and in clinical trials. $^{7818}$

In addition to reducing the burden of CHD for patients, it is also important that the financial burden of managing this disease be lowered. In the German healthcare system, cardiovascular disease accounts for the greatest costs directly attributable to disease. ${ }^{13}$ Although it was outside the scope of this study, the financial impact of alirocumab administration on patient treatment costs in Germany warrants further investigation.

Limitations of this study include its retrospective and observational design. The study was descriptive only, and no formal power calculation for any outcome was performed. The completeness of data may have varied between sites and there was the possibility of unmeasurable confounders being present. To minimise potential bias and reflect a broad cross-section of clinical experience, multiple sites were included. The observation period for alirocumab treatment was only 12 weeks, which limited the evaluation of effectiveness to the short-term only. The main strength of the study is that it provides information about how alirocumab is being prescribed in a real-world setting in Germany, and how this compares with German and European guidelines. Thus, the data are of potential interest to healthcare professionals and decision makers.

\section{CONCLUSIONS}

In conclusion, in a real-world setting in Germany, alirocumab was prescribed for patients with ASCVD who had high baseline LDL-C levels with/without statin intolerance. It was generally prescribed in compliance with national and internal guidelines. The results also suggest that treatment with alirocumab can provide effective lipid-lowering in real-world conditions. Efficacy and safety were consistent with findings observed in the ODYSSEY Phase III programme.

Contributors DS, PB, JM, CD, WDP and URK developed the concept of the current study. PB and JM collated all the data and PB wrote the first draft of the manuscript which all authors revised for important intellectual content. All authors gave final approval and agree to be accountable for all aspects of work ensuring integrity and accuracy. 
Funding This study was sponsored by Sanofi-Aventis Deutschland and funded by Sanofi and Regeneron Pharmaceuticals, Inc.

Competing interests PB and URK have received research support and/ or consulting honoraria from Amgen, Merck and Sanofi. JM, CD and WDP are employees of and are holding stock in Sanofi. DS, CS and MK have no potential conflict of interest to disclose.

Patient consent for publication Not required.

Provenance and peer review Not commissioned; externally peer reviewed.

Data availability statement Data are available upon reasonable request.

Open access This is an open access article distributed in accordance with the Creative Commons Attribution Non Commercial (CC BY-NC 4.0) license, which permits others to distribute, remix, adapt, build upon this work non-commercially, and license their derivative works on different terms, provided the original work is properly cited, appropriate credit is given, any changes made indicated, and the use is non-commercial. See: http://creativecommons.org/licenses/by-nc/4.0/.

ORCID iD

Peter Bramlage http://orcid.org/0000-0003-4970-2110

\section{REFERENCES}

1 Nichols M, Townsend N, Luengo-Fernandez R. European cardiovascular disease statistics 2012. European heart network, Brussels. European Society of Cardiology, Sophia Antipolis 2012

2 Moriarty PM, Thompson PD, Cannon CP, et al. Efficacy and safety of alirocumab vs ezetimibe in statin-intolerant patients, with a statin rechallenge arm: the odyssey alternative randomized trial. J Clin Lipidol 2015;9:758-69.

3 Benjamin EJ, Muntner P, Alonso A, et al. Heart disease and stroke Statistics-2019 update: a report from the American heart association. Circulation 2019;139:e56-28

4 Piepoli MF, Hoes AW, Agewall S, et al. 2016 European Guidelines on cardiovascular disease prevention in clinical practice: The Sixth Joint Task Force of the European Society of Cardiology and Other Societies on Cardiovascular Disease Prevention in Clinical Practice (constituted by representatives of 10 societies and by invited experts)Developed with the special contribution of the European Association for Cardiovascular Prevention \& Rehabilitation (EACPR). Eur Heart J 2016;37:2315-81.

5 Robinson JG, Rosenson RS, Farnier M, et al. Safety of Very Low Low-Density Lipoprotein Cholesterol Levels With Alirocumab: Pooled Data From Randomized Trials. J Am Coll Cardiol 2017;69:471-82.

6 Schwartz GG, Bessac L, Berdan LG, et al. Effect of alirocumab, a monoclonal antibody to PCSK9, on long-term cardiovascular outcomes following acute coronary syndromes: rationale and design of the ODYSSEY outcomes trial. Am Heart J 2014;168:682-9.

7 Sanofi-Aventis. Alirocumab (Praluent) summary of producr characteristics 2020

8 Schwartz GG, Steg PG, Szarek M, et al. Alirocumab and cardiovascular outcomes after acute coronary syndrome. $N$ Engl $J$ Med 2018:379:2097-107.

9 Landmesser U, Chapman MJ, Stock JK, et al. 2017 update of ESC/EAS Task Force on practical clinical guidance for proprotein convertase subtilisin/kexin type 9 inhibition in patients with atherosclerotic cardiovascular disease or in familial hypercholesterolaemia. Eur Heart J 2018;39:1131-43.

10 Mach F, Baigent C, Catapano AL, et al. 2019 ESC/EAS guidelines for the management of dyslipidaemias: lipid modification to reduce cardiovascular risk. Eur Heart J 2020;41:111-88.

11 Sanofi Aventis Deutschland GmbH. Prospektive nicht-interventionelle Studie zur optimierten Behandlung einer therapierefraktären heterozygot familiären oder nicht-familiären Hypercholesterinämie oder gemischter Dyslipidämie mit Alirocumab (PRALUENT®) bei Patienten mit gesicherter koronarer Herzkrankheit - (OPTIMIZE) 2018 Bundesinstitut für Arzneimittel und Medizinprodukte. Available: https://nebenwirkungen.bund.de/SharedDocs/awb/nis-0401-0500/ 0443.html [Accessed 11 Aug 2020].

12 Klose G, Laufs U, März W, et al. Familial hypercholesterolemia. Deutsches Aerzteblatt 2014

13 Dornquast C, Kroll LE, Neuhauser HK, et al. Regional differences in the prevalence of cardiovascular disease. Dtsch Arztebl Int 2016;113:704-11.

14 Khamis RY, Ammari T, Mikhail GW. Gender differences in coronary heart disease. Heart 2016;102:1142-9.

15 Maas AHEM, Appelman YEA. Gender differences in coronary heart disease. Neth Heart J 2010;18:598-603.

16 Allahyari A, Jernberg T, Hagström E, et al. Application of the 2019 ESC/EAS dyslipidaemia guidelines to nationwide data of patients with a recent myocardial infarction: a simulation study. Eur Heart $J$ 2020;41:3900-9.

17 Reynolds T, Carey P, George J, et al. A retrospective observational study to determine baseline characteristics and early prescribing patterns for patients receiving alirocumab in UK clinical practice. Drugs Real World Outcomes 2019;6:205-13.

18 Parhofer KG, von Stritzky B, Pietschmann N, et al. PEARL: a noninterventional study of real-world alirocumab use in German clinical practice. Drugs Real World Outcomes 2019;6:115-23.

19 Hollstein T, Kassner U, Grenkowitz T, et al. PCSK9 inhibitors in a German single-center clinical practice: real-world treatment of patients at high cardiovascular risk over 68 weeks. Am J Cardiovasc Drugs 2021;21:83-92.

20 Cordero A, Fácila L, Rodríguez-Mañero M, et al. Initial real-world experience with PCSK-9 inhibitors in current indications for reimbursement in Spain. Rev Esp Cardiol 2019;72:968-70. 